\title{
Mycorrhiza-induced resistance: more than the sum of its parts?
}

\author{
Duncan D. Cameron ${ }^{1}$, Andrew L. Neal ${ }^{2}$, Saskia C.M. van Wees ${ }^{3}$, and Jurriaan Ton ${ }^{1}$ \\ ${ }^{1}$ Department of Animal and Plant Sciences, University of Sheffield, Western Bank, Sheffield, S10 2TN, UK \\ ${ }^{2}$ Centre for Sustainable Soils and Grassland Systems, Rothamsted Research, Harpenden, AL5 2JQ, UK \\ ${ }^{3}$ Plant-Microbe Interactions, Department of Biology, Utrecht University, P.O. Box 800.56, 3508 TB Utrecht, The Netherlands
}

\begin{abstract}
Plants can develop an enhanced defensive capacity in response to infection by arbuscular mycorrhizal fungi (AMF). This 'mycorrhiza-induced resistance' (MIR) provides systemic protection against a wide range of attackers and shares characteristics with systemic acquired resistance (SAR) after pathogen infection and induced systemic resistance (ISR) following root colonisation by non-pathogenic rhizobacteria. It is commonly assumed that fungal stimulation of the plant immune system is solely responsible for MIR. In this opinion article, we present a novel model of MIR that integrates different aspects of the induced resistance phenomenon. We propose that MIR is a cumulative effect of direct plant responses to mycorrhizal infection and indirect immune responses to ISR-eliciting rhizobacteria in the mycorrhizosphere.
\end{abstract}

\section{Mycorrhiza-induced resistance (MIR)}

Mycorrhizal symbiosis is a mutualism between plants and mycorrhizal fungi during which photosynthetic products are exchanged for soil-derived mineral nutrients [1]. The true age of this relationship and the extent of host-mycorrhiza coevolution has been revealed by fossil evidence and phylogenetic analyses [2,3], dating the emergence of this symbiosis to 450 million years ago. It has been estimated that $80 \%$ of plant species retain these ancient arbuscular mycorrhizal associations [1], illustrating the importance of this mutualism to both partners.

Research on plant-mycorrhiza interactions has mostly focussed on the physiology of nutrient-for-carbon exchange and plant signal-transduction pathways controlling the interaction. Comparatively little is known about the mechanisms conferring non-nutritional benefits by mycorrhiza, such as suppression of soil-borne diseases and enhancing plant resistance to pests and diseases [4]. Plants routinely signal to conspecific organisms in the rhizosphere by releasing primary and secondary metabolites from their roots. Some of these metabolites recruit beneficial microbes, including AMF. Furthermore, AMF infection is known to stimulate biological activity in the rhizosphere, a phenomenon commonly referred to as the 'mycorrhizosphere effect' [5] (Box 1). This effect includes the attraction and selection of specific bacterial strains, such as plant

Corresponding author: Ton, J. (j.ton@sheffield.ac.uk).

$1360-1385 / \$$ - see front matter.

Crown Copyright (c) 2013 Published by Elsevier Ltd. All rights reserved. http:// dx.doi.org/10.1016/j.tplants.2013.06.004 growth-promoting rhizobacteria (PGPR) that possess the capacity to enhance plant growth and suppress pests and diseases. Some of these mycorrhizosphere-inhabiting bacteria can act as 'mycorrhiza-helper bacteria' and promote the efficiency of mycorrhizal symbiosis [6] (Box 1). As a consequence of these interactions, it has been suggested that the benefits of AMF on whole-plant physiology are at least partially determined by biological activities in the mycorrhizosphere [6-8].

AMF can suppress plant pests and diseases through induction of systemic resistance [9-11]. Nutrient supply experiments have revealed that MIR cannot be attributed to improved nutritional status [12]. The induced resistance shares characteristics with both pathogen-induced SAR and rhizobacterial ISR; MIR has been associated with SAR-like priming of salicylic acid (SA)-dependent genes, but more often coincides with priming of jasmonic acid (JA)-dependent defences and cell wall defences (Table 1). Accordingly, MIR confers protection against a wide range of attackers, including biotrophic pathogens, necrotrophic pathogens, nematodes, and herbivorous arthropods (Table 1). It has been proposed that MIR is the result of active suppression of components in the SA-dependent defence pathway, causing systemic priming of JA-dependent defences [10]. However, the exact contribution of jasmonates in MIR remains unclear [13] and the long-distance signals controlling MIR remain to be resolved. Most instances of MIR have been reported for non-sterile systems. It is thus possible that MIR is not solely determined by the fungus, but that bacteria in the mycorrhizosphere have a complementary contribution to the full MIR response. Here, we present a four-phase spatiotemporal model explaining MIR as a cumulative outcome of direct plant-AMF interactions and responses to ISR-eliciting bacteria in the mycorrhizosphere (Figure 1).

\section{Phase I: root exudation of mycorrhiza-recruiting} chemicals

Plant roots exude a diverse array of biologically active compounds [14]. Estimates suggest plants can exude up to $40 \%$ of their photosynthates from roots, representing a rich source of energy for soil microbes [15]. Root exudates typically contain sugars, amino acids, carboxylic acids, phenolics, and other secondary metabolites, which all have the capacity to influence the occurrence, physiology, and behaviour of soil organisms. For the interaction between plants and AMF, strigolactones have been identified as 


\section{Box 1. The mycorrhizosphere effect}

A relatively small volume of soil around plant roots is under the direct influence of root exudates, termed the 'rhizosphere'. This zone is characterised by increased levels of microbial activity. However, $80 \%$ of all plant species form symbiotic relationships with AMF [1]. Consequently, the volume of soil influenced by plant-derived carbon via AMF can be extended to encompass the 'mycorrhizosphere'. AMF have a selective influence on microbial communities in the mycorrhizosphere. The enhanced microbial activity surrounding mycorrhizal roots compared with non-mycorrhizal roots is called the 'mycorrhizosphere effect' [5]. Having resolved that extramatrical hyphae from AMF have access to resources from a vast volume of soil, it was discovered that some mycorrhizosphere-inhabiting bacteria, called 'mycorrhiza helper bacteria' (MHB), can stimulate mycorrhizal symbioses [6]. The concept of mutualism between AMF and soil bacteria is not new. In 1962, Mosse first proposed the idea that AMF and bacteria interact directly in the soil, showing that mycorrhizal roots can enhance the survival of $P$. fluorescens bacteria [74]. Since then, multiple studies have demonstrated that MHB can promote mycorrhizal infection and symbiosis through stimulation of mycelial extension and reducing the impact of adverse environmental

important AMF-recruiting signals (Box 2). This class of terpenoid lactones, long known as germination signals for parasitic plants, stimulates hyphal branching in AMF, thereby helping the fungus to localise host roots and so facilitate infection $[5,6]$. Involvement of root signals in the attraction of both pathogenic and mutualistic soil organisms is not rare. For instance, root-borne isoflavones secreted by soybean can attract the endosymbiotic N-fixing bacterium Bradyrhizobium japonicum [16], but can simultaneously attract the pathogenic oomycete Phytophthora sojae [17]. Similarly, benzoxazinoid metabolites in root exudates of maize (Zea mays) can attract both beneficial Pseudomonas putida bacteria [18] and western corn rootworm [19]. From an evolutionary perspective, common attraction of mutualistic and parasitic organisms is unsurprising given the strong selection pressure on parasites to adopt the same plant signals as beneficial mutualists (Box 2).

\section{Phase II: the plant immune system responding to AMF infection}

The initial stages of root colonisation by AMF are accompanied by transient induction of selected plant defences, followed by localised suppression at later stages of the interaction [20]. It is plausible that initial induction of plant immunity is based on host recognition of microbe-associated molecular patterns (MAMPs) from the AMF. Recognition of MAMPs by pattern-recognition receptors elicits a series of signalling cascades resulting in enhanced production of the plant defence hormone SA and expression of MAMP-triggered immunity [21]. For instance, infection by Funneliformis mosseae (syn. Glomus mosseae) induces transient accumulation of SA in pea [22], whereas this response was more pronounced and permanent in symbiosis-resistant P2 pea genotypes. Hence, the initial SA response is suppressed during successive stages of AMF infection. Localised MAMP recognition and SA production can lead to production of long-distance SAR signals and cause systemic priming of SA-dependent defences [23-25]. Because most SAR studies have been conducted with AMF-incompatible Arabidopsis, it is difficult to draw direct comparisons conditions [6]. Whether increased AMF growth and survival by MHB are due to production of growth factors, detoxification of soil allelochemicals, or antagonism of competitors and/or parasites remains unresolved [6]. With the development of metagenomics technologies and DNA-sequencing methods, the true extent of quantitative and qualitative changes in the microbial community due to AMF is beginning to emerge. The chemical basis driving mycorrhizosphere development is less well resolved, although there are indications that carbon exudation by AMF in the form of the glycoprotein glomalin plays a role [75]. This is an attractive hypothesis, given that up to $5 \%$ of active soil organic carbon pools comprise glomalin, which is recalcitrant in the soil and thus represents a 'slow-release' carbon substrate [75]. The consequences of the mycorrhizosphere effect, including recruitment of PGPRs, may not only boost nutrient mobilisation by AMF but could also provide non-nutritional benefits, such as disease suppression via antibiosis and/or competitive exclusion. Crucially, increased densities of selected rhizobacteria in the mycorrhizosphere have the potential to suppress pests and diseases in systemic plant tissues through priming of inducible defences.

between SAR and MIR. However, like SAR, MIR has been associated with systemic priming of SA-dependent defences and protection against (hemi)biotrophic pathogens (Table 1). Furthermore, the primed defence state of SAR is long lasting [26,27] and can act additively on other forms of systemic disease resistance [28]. We therefore propose that SAR-related signals during the early stages of plant-AMF interactions contribute to MIR (Figure 1).

\section{Phase III: immune suppression by AMF and recruitment of mycorrhizosphere bacteria}

The transient nature of MAMP-triggered immune responses during the early stages of mycorrhization suggest that AMF employ strategies similar to those of pathogenic fungi, which secrete specific effector molecules to suppress plant immunity and establish a successful infection [29]. A comparative transcriptome study in rice revealed striking similarities between responses to $\mathrm{AMF}$ and pathogenic fungi [30]. Additional evidence for active immune suppression by AMF came from the discovery that the calcium/calmodulin kinase DMI3, a central regulator in the symbiotic pathway, represses early-acting defence genes [31]. Kloppholz et al. [32] were the first to identify an effector protein (SP7) from Rhizophagus irregularis (syn. Glomus intraradices). This secreted protein is expressed during the initial stages of contact between the mycorrhizal fungus and roots and is translocated to the plant nucleus, where it inhibits the transcription factor ERF19 to suppress plant defence and promote infection by biotrophic fungi like $R$. irregularis [32]. AMF induce species-specific changes in defence hormones in their hosts [13,33]. Some of these hormonal changes can restrict AMF colonisation, whereas others function to promote biotrophic AMF infection. For instance, AMF promote production of the plant hormone abscisic acid (ABA) [34]. Experiments with the ABA-deficient tomato (Solanum lycopersicum) mutant sitiens have revealed that arbuscular development and functionality in tomato are dependent on ABA [35]. Because ABA can suppress SA-dependent defences against biotrophic pathogens [36,37], it is plausible that AMF stimulate ABA production in the roots to promote their 
Table 1. Defence mechanisms associated with ISR by AMF

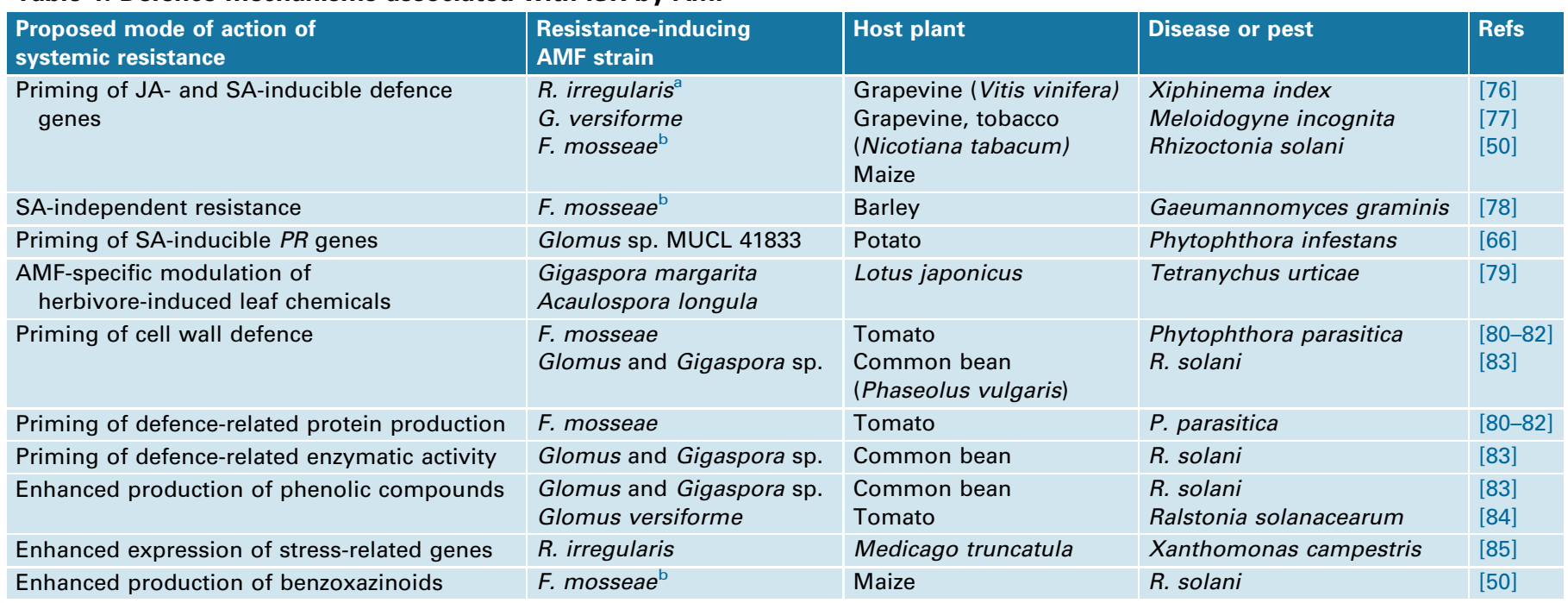

${ }^{\mathrm{a}}$ syn. G. intraradices.

${ }^{\mathrm{b}}$ syn. G. mosseae.

own infection. The role of ABA in disease resistance is complex and depends on the stage and nature of the interaction. Although ABA typically suppresses relatively late-acting defence mechanisms during plant-pathogen interactions (e.g., SA-dependent mechanisms), it can promote defence mechanisms that act relatively early in the interaction, such as MAMP-induced stomatal closure, induction of reactive oxygen species, and cell wall reinforcements [36]. The mobility of ABA through both xylem and phloem makes this hormone an attractive candidate to act as a complementary long-distance MIR signal to the shoot, where it could contribute to priming of cell wall defences (Figure 1). Indeed, application of ABA to maize roots induces above-ground resistance to the (hemi)necrotrophic fungi Setosphaeria turcicia and Colletotrichum graminicola $[38,39]$ and ABA treatment of rice enhances resistance against the necrotrophic fungus Cochliobolus miyabeanus [40]. However, shoot profiling of plant hormones in melon did not reveal consistent changes in ABA levels after root infection by either $G$. intraradices or $G$. mosseae [41], suggesting that a potential role for $\mathrm{ABA}$ as a systemic MIR signal may be transient.

Mycorrhization increases transport of photosynthates to the roots, influencing sugar-dependent signaling pathways
[42]. This, in combination with modulation of defence metabolism and improved phosphate uptake, leads to quantitative and qualitative changes in the composition of root exudates. For instance, AMF-mediated uptake of phosphorus suppresses strigolactone exudation [43], whereas other studies have reported quantitative and qualitative changes in primary metabolites from root exudates [44]. Some of these changes can have negative and positive impacts on other rhizosphere microbes [45-47]. Apart from plant-mediated changes in root exudate chemistry, metabolic activity by the fungus itself can also alter the chemical composition of mycorrhizal root exudates. Pulse-chase labeling experiments with ${ }^{13} \mathrm{CO}_{2}$ revealed that plant-assimilated carbon is transferred within hours to the fungus and can be traced back in specific mycorrhizosphere bacteria a few days later [47]. Exactly which AMF-induced changes in mycorrhizal root exudate chemistry shape the bacterial composition of the (mycor)rhizosphere remains difficult to predict on the basis of correlative studies and in vitro chemotaxis assays, but it is likely that a combination of primary and secondary metabolites is involved. In non-mycorrhizal Arabidopsis, mutation in the malate transporter gene ALMT1 affects recruitment of ISR-eliciting Bacillus subtilis FB17 after treatment of the leaves with MAMPs [48], indicating that

\section{Box 2. Strigolactones: multipurpose rhizosphere signals}

The strigolactones are a group of plant sesquiterpenes that are exuded from roots [86]. They serve as signals to induce hyphal branching of mycorrhizal fungi, leading to enhanced root colonisation by AMF $[87,88]$. However, 40 years ago, the same strigolactones were first identified as germination stimulants of parasitic plants from the Orobanchaceae family and the obligate root hemiparasitic plant Striga hermonthica (giant witchweed) [89], the first of these, strigol, being isolated from non-host cotton (Gossypium hirsutum) [89]. These observations generated a conundrum: why would plant roots produce signals to promote parasitic plant infection? Despite this unresolved issue, research continued to focus on understanding the biosynthesis of strigolactones and the diversity of Orobanchaceae species, for which they are able to induce germination. Consequently, until recently little was known about their additional beneficial role in the rhizosphere, despite the fact that non-hosts for parasitic Orobanchaceae produce strigolactones profusely. The breakthrough came from the observation that the synthetic strigolactone GR24 induces hyphal branching in in vitro cultures of the AMF Gigaspora margarita [87] and Gigaspora rosea [88]. Strigolactone-induced hyphal branching is thought to increase the probability of cellular contact between plant and fungus and, consequently, enhance root colonisation by AMF. The discovery of strigolactones as plant stimulants of mycorrhization demonstrates that parasitic plants have 'hijacked' an important host-symbiont signalling mechanism that predates their evolution by at least 200-300 million years [2,3]. To date, it remains unclear whether there are additional signals involved in the recruitment of AMF to plant roots. 


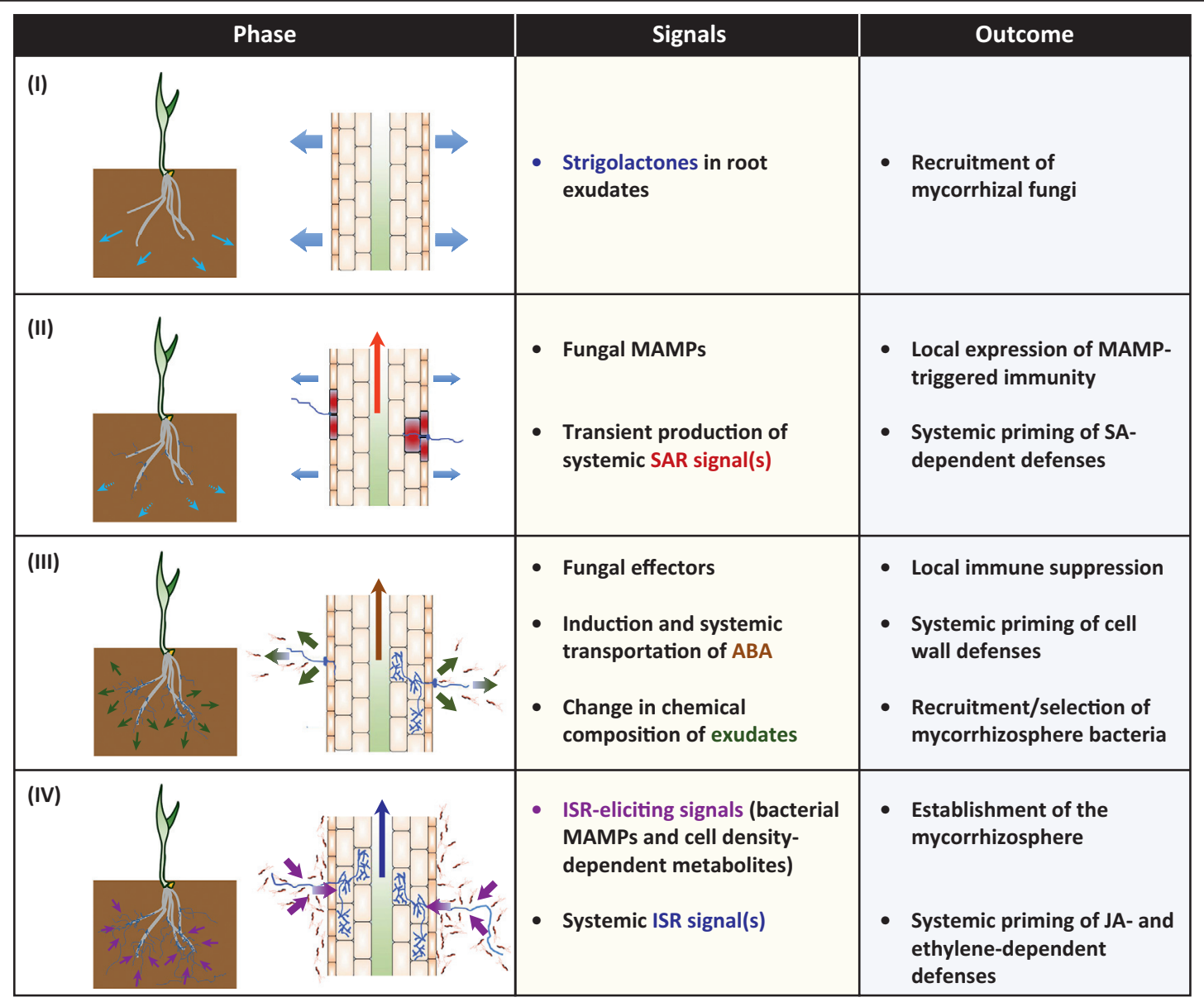

$\overline{\text { TRENDS in Plant Science }}$

Figure 1. Spatiotemporal model of mycorrhiza-induced resistance (MIR). Phase I: Root exudation of strigolactones (blue arrows) induces hyphal branching in arbuscular mycorrhizal fungi (AMF) and stimulates infection. Phase II: AMF initiate infection of the root cortex. Microbe-associated molecular patterns (MAMPs) from the fungus are recognised by the plant innate immune system. This leads to transient expression of MAMP-triggered immunity (red cells) and generation of long-distance signals in the

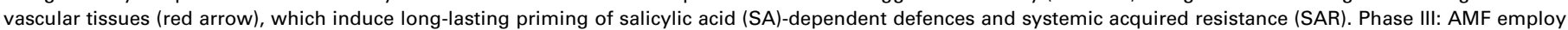
specific effector molecules and stimulate production of abscisic acid (ABA) to suppress MAMP-triggered immunity locally. ABA can be transported through the xylem to the shoot (brown arrow), where it can prime cell wall defences. Formation of intracellular arbuscules increases sugar import from the shoot and delivers phosphorous and other nutrients from the soil, thereby altering root metabolism and exudates. Moreover, metabolically active hyphae can alter the chemical composition of root exudates. The combined impact of plant immune modulation, enhanced sugar allocation, increased nutrient uptake, and fungal modification of root exudates leads to changes in root exudation chemistry (green arrows) and recruitment/selection of specific mycorrhizosphere bacteria. Phase IV: Establishment of the mycorrhizosphere is associated with dense colonisation by selected bacteria that metabolise mycorrhizal root exudates and deliver ISR-eliciting signals at the root surface and/or fungal hyphae (purple arrows). After perception of these signals by the host plant, long-distance signals (blue arrow) are generated that prime jasmonate- and ethylene-dependent plant defences and cause induced systemic resistance (ISR).

a single primary metabolite can be critical for recruitment of a specific rhizobacterial strain. There is also evidence for bacterial attraction by more complex secondary metabolites. Recently, it was found that mutation of the benzoxazinoid biosynthesis pathway in maize reduces attraction of ISReliciting $P$. putida KT2440 [18,49]. Interestingly, two additional studies have reported that infection of maize by the AMF $F$. mosseae or $R$. irregularis boosts production of root benzoxazinoids [50,51]. It is therefore tempting to speculate that AMF-induced exudation of a blend of benzoxazinoids contributes to cereal mycorrhizosphere development.

\section{Phase IV: establishment of the mycorrhizosphere and induction of systemic resistance by mycorrhizosphere bacteria}

Most MIR studies have quantified the level of resistance when the plant-AMF symbiosis and the mycorrhizosphere are fully established [9]. It is therefore possible that MIR involves an ISR component elicited by bacteria in the mycorrhizosphere (Figure 1). Like AMF, rhizobacteria possess MAMPs, which can trigger MAMP-induced immune responses [52]. Well-known examples of defenceeliciting MAMPs from bacteria are rhamnolipids, the elongation factor Tu, flagellin, and cell-wall lipopolysaccharides [53]. The spatially confined structure of the mycorrhizosphere allows rhizobacterial strains to reach exceptionally high cell densities [5]. Under these conditions, bacterial gene expression can be controlled by small diffusible signal molecules from members of the population themselves. This autoinduction process, known as quorum sensing (QS), allows bacteria to adjust community gene expression in accordance with their environment [54]. Many rhizosphere-colonizing bacteria, including Pseudomonas and Burkholderia strains, employ QS to control gene expression [55]. Some QS autoinducer molecules, like $N$-3-oxo-tetradecanoyl- $L$-homoserine lactone, can elicit 
resistance in Arabidopsis to Pseudomonas syringae and Golovinomyces orontii and in barley (Hordeum vulgare) to Blumeria graminis f. sp. hordei [56]. In addition to direct effects by autoinducer molecules, cell density-controlled processes in bacteria can also contribute to disease suppression. The PhzI/PhzR QS system in Pseudomonas chlororaphis mediates synthesis of the heterocyclic fungicide phenazine [57], whereas 2,4-diacetylphloroglucinol (2,4DAPG) produced by Pseudomonas fluorescens has autoinducer activity that can be counteracted by metabolites from other soil microbes [58]. Interestingly, both phenazine and 2,4-DAPG have been associated with ISR; expression of $P$. chlororaphis-mediated ISR in tobacco against necrotrophic Erwinia carotovora requires production of phenazine [59], whereas production of 2,4-DAPG by P. fluorescens is critical for JA-dependent ISR in Arabidopsis [60]. Considering that some bacterial strains reach sufficiently high cell densities in the mycorrhizosphere for autoinduction processes, it is plausible that cell density-dependent bacterial metabolites, like phenazine and 2,4-DAPG, contribute to MIR (Figure 1). Delivery of these ISR-eliciting determinants can be directly on the root surface, but can also be facilitated by efficient transportation into the root cortex through mycorrhizal hyphae [61]. Hence, the potential for mycorrhizosphere bacteria to elicit ISR is not only determined by their presence, but also depends on their metabolic activity in relation to chemical signals from mycorrhizal root exudates, their cell density, and the presence of competing microbes.

The nature of the systemic signals controlling rhizobacterial ISR is unknown. As for pathogen-induced SAR [62], ISR may be controlled by a combination of long-distance signals. Both ISR and MIR have frequently been associated with systemic priming of JA- and ethylene-inducible defences $[9,63,64]$. Jasmonates also accumulate during mycorrhizal symbiosis [13,33]. It is thus possible that jasmonates function as complementary long-distance signals of MIR, which may be the result of systemic signalling processes similar to autoregulation of nodulation during rhizobia-legume interactions [65]. Although the exact contribution of jasmonates to MIR has yet to be demonstrated, we propose that priming of JA-dependent defences during MIR is partially determined by ISR-eliciting rhizobacteria in the mycorrhizosphere (Figure 1).

\section{Concluding remarks and future research}

The concept that MIR is partially determined by resistanceinducing bacteria in the mycorrhizosphere creates a novel impetus to explore the complexity of biotic interactions and chemical signals surrounding mycorrhizal roots. The relative contribution of AMF and mycorrhizosphere-inhabiting bacteria to MIR requires experimental validation. To our knowledge, only one study has demonstrated MIR under strictly axenic conditions [66]. The induced resistance in this study was associated with augmented induction of two SA-inducible $P R$ genes following infection by Phytophthora infestans. Conversely, no clear transcriptional priming was evident for JA- and ethylene-dependent genes in this study [66], suggesting that axenic conditions prevent JA-dependent MIR. Whether priming of JA-dependent defences is strictly dependent on mycorrhizosphere-inhabiting bacteria would require complementation experiments with bacteria under axenic conditions. It is, however, possible that some ISReliciting mycorrhizosphere PGPR are not culturable and thrive only in close proximity to AMF hyphae [67]. It is even possible that endobacteria inside the AMF hyphae contribute to MIR [68]. A global inventory of microbial diversity through 16S RNA gene sequence analysis, coupled to temporal profiling of metabolites in mycorrhizal root exudates would be an alternative strategy to decipher the contribution of mycorrhizosphere bacteria in MIR. Involvement of candidate plant metabolites as regulators of resistance-inducing activities by mycorrhizosphere bacteria can be verified by genetic manipulation of the corresponding biosynthetic pathways in the host plant.

Further research is also required to elucidate the nature of systemic MIR signals. To determine whether selected plant hormones, such as ABA or jasmonates, act as longdistance signals in MIR would require grafting experiments with hormone-deficient plants. Unfortunately, interpretation of such experiments can be challenging, considering that hormone-deficient or -insensitive plant genotypes often develop stress phenotypes that can affect mycorrhizal symbiosis and complicate the interpretation of delicate plant-pathogen assays. In addition to plant hormones, small RNA molecules (sRNAs) are attractive candidates for long-distance defence signals. These 20-25nucleotide RNAs can act as phloem-mobile long-distance signals $[69,70]$. Moreover, small interfering (si)RNAs can induce transcriptional gene silencing through RNA-directed DNA methylation (RdDM) [71], a pathway that was recently implicated in transgenerational priming of JAand SA-dependent plant defence after exposure to herbivory and bacterial speck disease, respectively $[72,73]$. Global analysis of AMF-induced sRNAs and systemic changes in DNA methylation is needed to provide evidence for a possible contribution of sRNAs in the long-distance regulation of MIR. Finally, more comparative studies on the signalling mechanisms regulating MIR and ISR will be necessary to reveal the exact contribution of rhizobacteria to MIR.

\section{Acknowledgements}

J.T.'s research activities are supported by a grant from the European Research Council (ERC; no. 309944; 'PRIME-A-PLANT') and a Research Leadership Award from the Leverhulme Trust (no. RL-2012-042). D.D.C. is supported by a Royal Society University Research Fellowship. Rothamsted Research receives grant aid from the Biotechnology and Biological Science Research Council of the U.K. S.C.M.W.'s research is supported by the Dutch Technology Foundation STW (VIDI grant no. 11281), which is part of the Netherlands Organisation of Scientific Research. The authors also thank three anonymous reviewers for their helpful suggestions and constructive feedback on earlier versions of this article.

\section{References}

1 Smith, S.E. and Read, D.J. (2008) Mycorrhizal Symbiosis (3rd edn), Academic Press

2 Corradi, N. and Bonfante, P. (2012) The arbuscular mycorrhizal symbiosis: origin and evolution of a beneficial plant infection. PLoS Pathog. 8, e1002600

3 Field, K.J. et al. (2012) Contrasting arbuscular mycorrhizal responses of vascular and non-vascular plants to a simulated Palaeozoic $\mathrm{CO}_{2}$ decline. Nat. Commun. 3, 835

4 Cameron, D. (2010) Arbuscular mycorrhizal fungi as (agro)ecosystem engineers. Plant Soil 333, 1-5 
5 Linderman, R.G. (1988) Mycorrhizal Interactions with the rhizosphere microflora-the mycorrhizosphere effect. Phytopathology 78, 366-371

6 Frey-Klett, P. et al. (2007) The mycorrhiza helper bacteria revisited. New Phytol. 176, 22-36

7 Bending, G.D. et al. (2006) Significance of microbial interactions in the mycorrhizosphere. Adv. Appl. Microbiol. 60, 97-132

8 Johansson, J.F. et al. (2004) Microbial interactions in the mycorrhizosphere and their significance for sustainable agriculture. FEMS Microbiol. Ecol. 48, 1-13

9 Jung, S.C. et al. (2012) Mycorrhiza-induced resistance and priming of plant defenses. J. Chem. Ecol. 38, 651-664

10 Pozo, M.J. and Azcon-Aguilar, C. (2007) Unraveling mycorrhizainduced resistance. Curr. Opin. Plant Biol. 10, 393-398

11 Pineda, A. et al. (2010) Helping plants to deal with insects: the role of beneficial soil-borne microbes. Trends Plant Sci. 15, 507-514

12 Fritz, M. et al. (2006) Arbuscular mycorrhiza reduces susceptibility of tomato to Alternaria solani. Mycorrhiza 16, 413-419

13 Hause, B. et al. (2007) Jasmonates in arbuscular mycorrhizal interactions. Phytochemistry 68, 101-110

14 Bais, H.P. et al. (2006) The role of root exudates in rhizosphere interations with plants and other organisms. Annu. Rev. Plant Biol. $57,233-266$

15 Lugtenberg, B.J. et al. (1999) Tomato seed and root exudate sugars: composition, utilization by Pseudomonas biocontrol strains and role in rhizosphere colonization. Environ. Microbiol. 1, 439-446

16 Subramanian, S. et al. (2007) Distinct, crucial roles of flavonoids during legume nodulation. Trends Plant Sci. 12, 282-285

17 Morris, P.F. et al. (1998) Chemotropic and contact responses of Phytophthora sojae hyphae to soybean isoflavonoids and artificial substrates. Plant Physiol. 117, 1171-1178

18 Neal, A.L. et al. (2012) Benzoxazinoids in root exudates of maize attract Pseudomonas putida to the rhizosphere. PLoS ONE 7, e35498

19 Robert, C.A.M. et al. (2012) A specialist root herbivore exploits defensive metabolites to locate nutritious tissues. Ecol. Lett. 15, 55-64

20 Kapulnik, Y. et al. (1996) Suppression of defence responses in mycorrhizal alfalfa and tobacco roots. New Phytol. 133, 59-64

21 Zhang, J. and Zhou, J.M. (2010) Plant immunity triggered by microbial molecular signatures. Mol. Plant 3, 783-793

22 Blilou, I. et al. (1999) Resistance of pea roots to endomycorrhizal fungus or Rhizobium correlates with enhanced levels of endogenous salicylic acid. J. Exp. Bot. 50, 1663-1668

23 Conrath, U. (2011) Molecular aspects of defence priming. Trends Plant Sci. $16,524-531$

24 Heil, M. and Ton, J. (2008) Long-distance signalling in plant defence. Trends Plant Sci. 13, 264-272

25 Mishina, T.E. and Zeier, J. (2007) Pathogen-associated molecular pattern recognition rather than development of tissue necrosis contributes to bacterial induction of systemic acquired resistance in Arabidopsis. Plant J. 50, 500-513

26 Luna, E. et al. (2012) Next-generation systemic acquired resistance. Plant Physiol. 158, 844-853

27 Slaughter, A. et al. (2012) Descendants of primed Arabidopsis plants exhibit resistance to biotic stress. Plant Physiol. 158, 835-843

28 Van Wees, S.C.M. et al. (2000) Enhancement of induced disease resistance by simultaneous activation of salicylate- and jasmonatedependent defense pathways in Arabidopsis thaliana. Proc. Natl. Acad. Sci. U.S.A. 97, 8711-8716

29 de Jonge, R. et al. (2011) How filamentous pathogens co-opt plants: the ins and outs of fungal effectors. Curr. Opin. Plant Biol. 14, 400-406

30 Guimil, S. et al. (2005) Comparative transcriptomics of rice reveals an ancient pattern of response to microbial colonization. Proc. Natl. Acad. Sci. U.S.A. 102, 8066-8070

31 Siciliano, V. et al. (2007) Transcriptome analysis of arbuscular mycorrhizal roots during development of the prepenetration apparatus. Plant Physiol. 144, 1455-1466

32 Kloppholz, S. et al. (2011) A secreted fungal effector of Glomus intraradices promotes symbiotic biotrophy. Curr. Biol. 21, 1204-1209

33 Lopez-Raez, J.A. et al. (2010) Hormonal and transcriptional profiles highlight common and differential host responses to arbuscular mycorrhizal fungi and the regulation of the oxylipin pathway. $J$ Exp. Biol. 61, 2589-2601

34 Bothe, H. et al. (1994) Biochemical approaches to the study of plantfungal interactions in arbuscular mycorrhiza. Experientia 50, 919-925
35 Herrera-Medina, M.J. et al. (2007) Abscisic acid determines arbuscule development and functionality in the tomato arbuscular mycorrhiza. New Phytol. 175, 554-564

36 Ton, J. et al. (2009) The multifaceted role of ABA in disease resistance. Trends Plant Sci. 14, 310-317

37 Asselbergh, B. et al. (2008) Global switches and fine-tuning-ABA modulates plant pathogen defense. Mol. Plant Microbe Interact. 21, 709-719

$38 \mathrm{Erb}$, M. et al. (2009) Signal signature of aboveground-induced resistance upon belowground herbivory in maize. Plant J. 59, 292-302

39 Balmer, D. et al. (2013) Induced resistance in maize is based on organspecific defence responses. Plant J. http://dx.doi.org/10.1111/tpj.12114

40 De Vleesschauwer, D. et al. (2010) Abscisic acid-induced resistance against the brown spot pathogen Cochliobolus miyabeanus in rice involves MAP kinase-mediated repression of ethylene signaling. Plant Physiol. 152, 2036-2052

41 Martinez-Medina, A. et al. (2011) The interaction with arbuscular mycorrhizal fungi or Trichoderma harzianum alters the shoot hormonal profile in melon plants. Phytochemistry 72, 223-229

42 Smith, S.E. and Smith, F.A. (2011) Roles of arbuscular mycorrhizas in plant nutrition and growth: new paradigms from cellular to ecosystem scales. Annu. Rev. Plant Biol. 62, 227-250

43 Bouwmeester, H.J. et al. (2007) Rhizosphere communication of plants, parasitic plants and AM fungi. Trends Plant Sci. 12, 224-230

44 Graham, J.H. et al. (1981) Membrane-mediated decrease in root exudation responsible for phorphorus inhibition of vesiculararbuscular mycorrhiza formation. Plant Physiol. 68, 548-552

45 Marschner, P. et al. (1997) Root exudation and physiological status of a root-colonizing fluorescent pseudomonad in mycorrhizal and nonmycorrhizal pepper (Capsicum annuum L.). Plant Soil 189, 11-20

46 Gupta Sood, S. (2003) Chemotactic response of plant-growthpromoting bacteria towards roots of vesicular-arbuscular mycorrhizal tomato plants. FEMS Microbiol. Ecol. 45, 219-227

47 Drigo, B. et al. (2010) Shifting carbon flow from roots into associated microbial communities in response to elevated atmospheric $\mathrm{CO}_{2}$. Proc. Natl. Acad. Sci. U.S.A. 107, 10938-10942

48 Lakshmanan, V. et al. (2012) Microbe-associated molecular patternstriggered root responses mediate beneficial rhizobacterial recruitment in Arabidopsis. Plant Physiol. 160, 1642-1661

49 Neal, A. and Ton, J. (2012) Systemic defense priming by Pseudomonas putida KT2440 in maize depends on benzoxazinoid exudation from the roots. Plant Signal. Behav. 8, e22655

50 Song, Y. et al. (2011) Induction of DIMBOA accumulation and systemic defense responses as a mechanism of enhanced resistance of mycorrhizal corn (Zea mays) to sheath blight. Mycorrhiza 21, 721-731

51 Walker, V. et al. (2012) Variation of secondary metabolite levels in maize seedling roots induced by inoculation with Azospirillum, Pseudomonas and Glomus consortium under field conditions. Plant Soil 356, 151-163

52 Berendsen, R.L. et al. (2012) The rhizosphere microbiome and plant health. Trends Plant Sci. 17, 478-486

53 Boller, T. and Felix, G. (2009) A renaissance of elicitors: perception of microbe-associated molecular patterns and danger signals by patternrecognition receptors. Annu. Rev. Plant Biol. 60, 379-406

54 Waters, C.M. and Bassler, B.L. (2005) Quorum sensing: cell-to-cell communication in bacteria. Annu. Rev. Cell Dev. Biol. 21, 319-346

55 Lugtenberg, B. and Kamilova, F. (2009) Plant-growth-promoting rhizobacteria. Annu. Rev. Microbiol. 63, 541-556

56 Schikora, A. et al. (2011) N-acyl-homoserine lactone confers resistance toward biotrophic and hemibiotrophic pathogens via altered activation of AtMPK6. Plant Physiol. 157, 1407-1418

57 Selin, C. et al. (2012) The PhzI/PhzR quorum-sensing system is required for pyrrolnitrin and phenazine production, and exhibits cross-regulation with RpoS in Pseudomonas chlororaphis PA23. Microbiology 158, 896-907

58 Schnider-Keel, U. et al. (2000) Autoinduction of 2,4diacetylphloroglucinol biosynthesis in the biocontrol agent Pseudomonas fluorescens CHAO and repression by the bacterial metabolites salicylate and pyoluteorin. J. Bacteriol. 182, 1215-1225

59 Kang, B.R. et al. (2007) Inhibition of seed germination and induction of systemic disease resistance by Pseudomonas chlororaphis $\mathrm{O} 6$ requires phenazine production regulated by the global regulator, gacS. J. Microbiol. Biotechnol. 17, 586-593 
60 Iavicoli, A. et al. (2003) Induced systemic resistance in Arabidopsis thaliana in response to root inoculation with Pseudomonas fluorescens CHA0. Mol. Plant Microbe Interact. 16, 851-858

61 Barto, E.K. et al. (2012) Fungal superhighways: do common mycorrhizal networks enhance below ground communication? Trends Plant Sci. 17, 633-637

62 Dempsey, D.A. and Klessig, D.F. (2012) SOS-too many signals for systemic acquired resistance? Trends Plant Sci. 17, 538-545

63 Van der Ent, S. et al. (2009) Jasmonate signaling in plant interactions with resistance-inducing beneficial microbes. Phytochemistry 70, 1581-1588

64 Van Wees, S.C. et al. (2008) Plant immune responses triggered by beneficial microbes. Curr. Opin. Plant Biol. 11, 443-448

65 Zamioudis, C. and Pieterse, C.M. (2012) Modulation of host immunity by beneficial microbes. Mol. Plant Microbe Interact. 25, 139-150

66 Gallou, A. et al. (2011) Mycorrhiza induced resistance in potato plantlets challenged by Phytophthora infestans. Physiol. Mol. Plant Pathol. 76, 20-26

67 Scheublin, T.R. et al. (2010) Characterisation of microbial communities colonising the hyphal surfaces of arbuscular mycorrhizal fungi. ISME J. 4, 752-763

68 Bonfante, P. and Anca, I.A. (2009) Plants, mycorrhizal fungi, and bacteria: a network of interactions. Annu. Rev. Microbiol. 63, 363-383

$69 \mathrm{Kehr}$, J. and Buhtz, A. (2008) Long distance transport and movement of RNA through the phloem. J. Exp. Bot. 59, 85-92

70 Dunoyer, P. et al. (2010) Small RNA duplexes function as mobile silencing signals between plant cells. Science 328, 912-916

71 Zhang, H. and Zhu, J.K. (2011) RNA-directed DNA methylation. Curr. Opin. Plant Biol. 14, 142-147

72 Rasmann, S. et al. (2012) Herbivory in the previous generation primes plants for enhanced insect resistance. Plant Physiol. 158, 854-863

73 Luna, E. and Ton, J. (2012) The epigenetic machinery controlling transgenerational systemic acquired resistance. Plant Signal. Behav. 7, 615-618

74 Mosse, B. (1962) The establishment of vesicular-arbuscular mycorrhiza under aseptic conditions. J. Gen. Microbiol. 27, 509-520

75 Rillig, M.C. et al. (2003) Glomalin, an arbuscular-mycorrhizal fungal soil protein, responds to land-use change. Plant Soil 253, 293-299

76 Hao, Z. et al. (2012) Local and systemic mycorrhiza-induced protection against the ectoparasitic nematode Xiphinema index involves priming of defence gene responses in grapevine. J. Exp. Biol. 63, 3657-3672
77 Li, H.Y. et al. (2006) Colonization by the arbuscular mycorrhizal fungus Glomus versiforme induces a defense response against the root-knot nematode Meloidogyne incognita in the grapevine (Vitis amurensis Rupr.), which includes transcriptional activation of the class III chitinase gene VCH3. Plant Cell Physiol. 47, 154-163

78 Khaosaad, T. et al. (2007) Take-all disease is systemically reduced in roots of mycorrhizal barley plants. Soil Biol. Biochem. 39, $727-734$

79 Nishida, T. et al. (2010) Arbuscular mycorrhizal fungi speciesspecifically affect induced plant responses to a spider mite. Popul. Ecol. 52, 507-515

80 Cordier, C. et al. (1998) Cell defense responses associated with localized and systemic resistance to Phytophthora induced in tomato by an arbuscular mycorrhizal fungus. Mol. Plant Microbe Interact. 11, 1017-1028

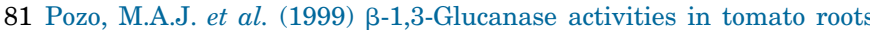
inoculated with arbuscular mycorrhizal fungi and/or Phytophthora parasitica and their possible involvement in bioprotection. Plant Sci. $141,149-157$

82 Pozo, M.J. et al. (2002) Localized vs systemic effect of arbuscular mycorrhizal fungi on defence responses to Phytophthora infection in tomato plants. J. Exp. Bot. 53, 525-534

83 Abdel-Fattah, G.M. et al. (2011) Induction of defense responses in common bean plants by arbuscular mycorrhizal fungi. Microbiol. Res. 166, 268-281

$84 \mathrm{Zhu}, \mathrm{H} . \mathrm{H}$. and Yao, Q. (2004) Localized and systemic increase of phenols in tomato roots induced by Glomus versiforme inhibits Ralstonia solanacearum. J. Phytopathol. 152, 537-542

85 Liu, J. et al. (2007) Arbuscular mycorrhizal symbiosis is accompanied by local and systemic alterations in gene expression and an increase in disease resistance in the shoots. Plant J. 50, 529-544

86 Xie, X. and Yoneyama, K. (2010) The strigolactone story. Annu. Rev. Phytopathol. 48, 93-117

87 Akiyama, K. et al. (2005) Plant sesquiterpenes induce hyphal branching in arbuscular mycorrhizal fungi. Nature 435, 824-827

88 Besserer, A. et al. (2006) Strigolactones stimulate arbuscular mycorrhizal fungi by activating mitochondria. PLoS Biol. 4, e226

89 Cook, C.E.et al. (1966) Germination of witchweed (Striga lutea Lour.): isolation and properties of a potent stimulant. Science 154, 1189-1190

\title{
Plant Science Conferences in 2013
}

\author{
The Plant Genomics Congress USA \\ 23-24 September, 2013 \\ St. Louis, USA \\ http://www.globalengage.co.uk/plantgenomicsusa.html \\ The 11th International Conference on Plant Anaerobiosis \\ 6-11 October, 2013 \\ Los Baños, Philippines \\ http://www.ispa2013.org/
}

6th European Workshop on Leaf Senescence

14-18 October 2013

Versailles, France

https://colloque.inra.fr/6th_workshop_on_leaf_senescence 